\title{
86. Recurrence of a Diffusion Process in a Multidimensional Brownian Environment*)
}

\author{
By Hiroshi TANAKA \\ Department of Mathematics, Faculty of Science and Technology, Keio University \\ (Communicated by Kiyosi ITÔ, M. J. A., Nov. 12, 1993)
}

Introduction. Let $\boldsymbol{W}$ be the space of continuous functions on $\boldsymbol{R}^{d}$ vanishing at the origin. In this paper an element of $\boldsymbol{W}$ is called an environment. Given an environment $\boldsymbol{W}$, we consider a diffusion process $\boldsymbol{X}_{W}=$ $\left\{X(t), t \geq 0, P_{W}^{x}, x \in \boldsymbol{R}^{d}\right\}$ with generator

$$
\frac{1}{2}(\Delta-\nabla W \cdot \nabla)=\frac{1}{2} e^{W} \sum_{k=1}^{d} \frac{\partial}{\partial x_{k}}\left(e^{-W} \frac{\partial}{\partial x_{k}}\right) .
$$

When $W$ is bounded, the result of Nash [8] for fundamental solutions of parabolic equations guarantees the existence of a diffusion process $\boldsymbol{X}_{W}^{0}$ with generator

$$
\sum_{k=1}^{d} \frac{\partial}{\partial x_{k}}\left(e^{-W} \frac{\partial}{\partial x_{k}}\right)
$$

For a general $W$ we still have a nice diffusion process $\boldsymbol{X}_{W}^{0}$ (e.g. see [4]) and hence $\boldsymbol{X}_{W}$ can be constructed from $\boldsymbol{X}_{W}^{0}$ through a random time change. Without any assumption on the behavior of $W(x)$ for large $|x|$ the process $\boldsymbol{X}_{W}$ may explode within a finite time, but such a case is excluded automatically since we are interested in the recurrence of $\boldsymbol{X}_{W}$. We consider the probability measure $\boldsymbol{P}$ on $\boldsymbol{W}$ with respect to which $\left\{W(x), x \in \boldsymbol{R}^{d}, P\right\}$ is a Lévy's Brownian motion with a $d$-dimensional time. The collection of diffusion processes $\boldsymbol{X}=\left\{\boldsymbol{X}_{W}\right\}$ in which $W$ is allowed to vary as a random element in $(\boldsymbol{W}, P)$ is called a diffusion in a $d$-dimensional Brownian environment. When $d=1$ this was considered by Brox [1] and Schumacher [9] as a diffusion model exhibiting the same asymptotic behavior as Sinai's random walk in a random environment ([10]); see also [11] for some refined results. Recently Mathieu [7] obtained some very interesting results concerning a long time asymptotic problem for $\boldsymbol{X}$ in the case $d \geq 2$. Motivated by [7] the present paper was written.

In this paper we prove that $\boldsymbol{X}_{W}$ is recurrent for almost all Brownian environments $W$ in any dimension $d$, namely, for any nonnegative Borel function $f$ on $\boldsymbol{R}^{d}$ such that $f>0$ on a set of positive Lebesgue measure the equality

$$
P_{W}^{x}\left\{\int_{0}^{\infty} f(X(t)) d t=\infty\right\}=1, x \in \boldsymbol{R}^{d}
$$

holds for almost all $W$ with respect to $P$. In [3] Fukushima, Nakao and Takeda discussed the same problem but with the replacement of $W(x)$ by $\tilde{W}(|x|)$,

*) This research was partially supported by Grant-in-Aid for Science Research No. 04452011, the Ministry of Education, Science and Culture, Japan. 
where $\tilde{W}(t)$ is a Brownian motion with a 1 -dimensional time.

To obtain our result we employ Ichihara's recurrence criterion ([4]) which, in the present special case, asserts that $\boldsymbol{X}_{W}^{0}$ (W is fixed) is recurrent if

$$
\int_{1}^{\infty}\left\{\int_{S^{d-1}} e^{-W(r \theta)} d \theta\right\}^{-1} r^{-d+1} d r=\infty
$$

where $d \theta$ is the uniform distribution on $S^{d-1}$. We can also employ Fukushima's recurrence criterion ([2]) which, in the present special case, asserts that $\boldsymbol{X}_{W}\left(W\right.$ is fixed) is recurrent if there exists a sequence $\left\{u_{n}\right\}$ such that $0 \leq u_{n}$ $\leq 1, \lim u_{n}=1$ a.e. and $\lim \mathscr{E}\left(u_{n}, u_{n}\right)=0$, where $\mathscr{E}(u, v)$ is the Dirichlet form associated with $\boldsymbol{X}_{W}$, namely,

$$
\mathscr{E}(u, v)=\frac{1}{2} \int_{\boldsymbol{R}^{d}} \nabla u \cdot \nabla u e^{-W} d x .
$$

Since it is obvious that $\boldsymbol{X}_{W}$ is recurrent if and only if $\boldsymbol{X}_{W}^{0}$ is recurrent, either criterion yields our result. But for the verification of these criteria we need some information on the asymptotic behavior of $W(x)$ for large $|x|$. A key point in obtaining this informaiton is to consider the one-parameter family $\left\{T_{t}, t \in \boldsymbol{R}\right\}$ of measure preserving transformations on $(\boldsymbol{W}, P)$ defined by (1. 3 ) and then to use its ergodicity.

\$1. Brownian motion with a $d$-dimensional time. Let $d \geq 2$ and as before let $P$ be the probability measure on $\boldsymbol{W}$ such that $\left\{W(x), x \in \boldsymbol{R}^{d}, P\right\}$ is a Brownian motion with a $d$-dimensional time ([6: p. 277]), that is, a Gaussian system with

$$
\begin{gathered}
E\{W(x)\}=0, W(0)=0, \\
E\{W(x) W(y)\}=\frac{1}{2}\{|x|+|y|-|x-y|\} .
\end{gathered}
$$

For each $t \in \boldsymbol{R}$ and $W \in \boldsymbol{W}$ we define an element $T_{t} W$ of $\boldsymbol{W}$ by

$$
\left(T_{t} W\right)(x)=e^{-t / 2} W\left(e^{t} x\right), \quad x \in \boldsymbol{R}^{d} .
$$

Then $\left\{T_{t}, t \in \boldsymbol{R}\right\}$ is a one-parameter family of measure preserving transformations on the probalility space $(\boldsymbol{W}, P)$. Using (1. 2) we can easily compute the covariance matrix of

$$
e^{-t / 2} W\left(e^{t} x_{1}\right), e^{-t / 2} W\left(e^{t} x_{2}\right), \cdots, e^{-t / 2} W\left(e^{t} x_{m}\right), W\left(x_{1}^{\prime}\right), W\left(x_{2}^{\prime}\right), \cdots, W\left(x_{n}^{\prime}\right)
$$

for fixed $t \in \boldsymbol{R}$ and $\mathrm{x}_{1}, \ldots, x_{m}, x_{1}^{\prime}, \ldots, x_{n}^{\prime} \in \boldsymbol{R}^{d}$, and the following lemma can be proved in the same way as in Itô [5].

Lemma 1. $\left\{T_{t}, t \in \boldsymbol{R}\right\}$ is mixing and hence ergodic.

Next let $0<a<b$, put $K=\left\{x \in \boldsymbol{R}^{d}: a \leq|x| \leq b\right\}$ and consider the Banach space $B=C(K)$, the space of real valued continuous functions on $K$, and the real Hilbert space $H=L^{2}(K, d x)$. The inner product in $H$ is denoted by $\langle\cdot, \cdot\rangle$. Regarding $W_{K}=\{W(x), x \in K\}$ as an $H$-valued random variable, we denote by $\gamma$ the probability distribution of $W_{K}$. Since every Borel set in the space $B$ is also a Borel set in the space $H$ and since $W_{K}$ is regarded as a $B$-valued random variable, we have $\gamma(B)=1 . \gamma$ is a Gaussian measure on $H$ with

$$
\text { (1.4a) } \int_{H} e^{\langle f, g\rangle} \gamma(d g)=E\left\{\exp \int_{K} f(x) W(x) d x\right\}=\exp \left\{\frac{1}{2}\langle A f, f\rangle\right\}, f \in H,
$$




$$
A f(x)=\int_{K} \frac{1}{2}\{|x|+|y|-|x-y|\} f(y) d y .
$$

For $\phi=A f_{0}$ with $f_{0} \in H$ we define the $\phi$-transform $\gamma_{\phi}$ by $\gamma_{\phi}(\Gamma)=\gamma(\{g: g$ $+\phi \in \Gamma\})$. Then the following Cameron-Martin formula is easily verified by using (1. 4).

$$
\gamma_{\phi}(d g)=\exp \left\{\left\langle f_{0}, g\right\rangle-\frac{1}{2}\left\langle A f_{0}, f_{0}\right\rangle\right\} \gamma(d g) .
$$

Lemma 2. Any nonempty open set in the space $B$ has a positive $\gamma$-measure.

Proof. We first prove that the range $R=\{A f: f \in H\}$ is dense in $B$. If this were not true, there exists a finite signed measure $\mu \neq 0$ on $K$ such that

$$
\int_{K} A f(x) \mu(d x)=0 \text { for all } f \in H .
$$

Since the left hand side of (1.6) equals $\langle f, g\rangle$ where

$$
g(x)=\int_{K} \frac{1}{2}\{|x|+|y|-|x-y|\} \mu(d y) \in H,
$$

(1.6) implies $g=0$. Therefore, regarding $\mu$ as a signed measure in $\boldsymbol{R}^{d}$ we have

$$
\int_{\boldsymbol{R}^{d}} \int_{\boldsymbol{R}^{d}} \frac{1}{2}\{|x|+|y|-|x-y|\} \mu(d y) \mu(d y)=0 .
$$

In the same way as in the proof of Theorème 58 of [6: p. 276] we can prove that the left hand side of (1.7) equals

$$
\text { const. } \int_{\boldsymbol{R}^{d}}|\xi|^{-d-1}|\hat{\mu}(\xi)-\hat{\mu}(0)|^{2} d \xi,
$$

where $\hat{\mu}(\xi)$ is the Fourier transform of $\mu$. Therefore $\mu$ must be concentrated on $\{0\}$. But this is impossible because $0 \notin K$ and hence $R$ must be dense in $B$. Next we notice that the whole space $B$, which has $\gamma$-measure 1 , can be expresses as a union of a countable number of open balls of the form $B_{\varepsilon}(\phi)=\left\{\phi \in B:\|\phi-\phi\|_{\infty}<\varepsilon\right\}, \phi \in R, \varepsilon>0$ being arbitrary but fixed. On the other hand by the Cameron-Martin formula (1.5) $r\left(B_{\varepsilon}(\phi)\right)=$ $\gamma_{-\phi}\left(B_{\varepsilon}(0)\right)>0$ if and only if $\gamma\left(B_{\varepsilon}(0)\right)>0$ provided that $\phi \in R$. Therefore we must have $\gamma\left(B_{\varepsilon}(\phi)\right)>0$ for all $\phi \in R$. This implies the assertion of the lemma.

§2. Recurrence of $\boldsymbol{X}_{W}$. Since our result in the 1 -dimensional case is easily obtained from a general theory of 1-dimensional diffusion processes, we assume $d \geq 2$.

Theorem 1. $\boldsymbol{X}_{W}$ is recurrent for almost all Brownian environments $W$.

Proof. It is enough to prove that $\boldsymbol{X}_{W}^{0}$ is recurrent for almost all Brownian environments $W$ and, according to Ichihara's criterion ([4: Theorem A]) it is also enough to prove that

$$
\int_{1}^{\infty}\left\{\int_{S^{d-1}} e^{-W(r \theta)} d \theta\right\}^{-1} r^{-d+1} d r=\infty, P-a . s .
$$

If we put $M(t)=\min \left\{\left(T_{t} W\right)(\theta): \theta \in S^{d-1}\right\}$, then

$$
\text { the left hand side of (2.1) }
$$




$$
\begin{aligned}
& =\int_{0}^{\infty} e^{(2-d) t}\left\{\int_{S^{d-1}} \exp \left(-e^{t / 2}\left(T_{t} W\right)(\theta)\right) d \theta\right\}^{-1} d t \\
\geq & \int_{0}^{\infty} e^{(2-d) t} \exp \left\{e^{t / 2} M(t)\right\} d t \geq \int_{0}^{\infty} \mathbf{1}_{(a, \infty)}(M(t)) d t,
\end{aligned}
$$

provided that $a>0$ is chosen so that $(2-d) t+a e^{t / 2} \geq 0$ holds for all $t \geq$ 0 . Next take $K=\left\{x \in \boldsymbol{R}^{d}: 1 \leq|x| \leq 2\right\}$ and consider $B, H$ and $\gamma$ as in the preceeding section. Since $\Gamma=\{\phi \in B: \min (\phi(x):|x|=1)>a\}$ is an open set in $B$, we have $\gamma(\Gamma)>0$ by Lemma 2 . The ergodicity of $\left\{T_{t}, t \in \boldsymbol{R}\right\}$ now implies

$$
\lim _{T \rightarrow \infty} T^{-1} \int_{0}^{T} \mathbf{1}_{(a, \infty)}(M(t)) d t=E\left\{\mathbf{1}_{(a, \infty)}(M(0))\right\}=r(\Gamma)>0, P-a, s .,
$$

and hence $\int_{0}^{\infty} \mathbf{1}_{(a, \infty)}(M(t)) d t=\infty, P$-a.s., which combined with proves (2. 1).

Remark 1. $\boldsymbol{X}_{W}$ is null-recurrent $(P-a . s$.$) in the sense that m_{W}(d x)=$ $e^{-W} d x$ is an invariant measure for $\boldsymbol{X}_{W}$ with $m_{W}\left(\boldsymbol{R}^{d}\right)=\infty$.

Remark 2. Fukushima's criterion can also be used for proving Theorem 1; in fact, by virtue of Lemmas 1,2 it is still easy to prove the existence of a sequence of radial functions $u_{n}$ in $C_{0}^{\infty}\left(\boldsymbol{R}^{d}\right)$ such that $0 \leq u_{n}$ $\leq 1, \lim u_{n}=1$ a.e. and $\mathscr{E}\left(u_{n}, u_{n}\right)=0$. This argument also proves the recurrence of $\boldsymbol{X}_{|W|}$ for almost all Brownian environments $W$.

Remark 3. $\boldsymbol{X}_{-|W|}$ is recurrent for $d=1$ and transient for $d \geq 2$ for almost all Brownian environments $W$. The proof in the case $d \geq 2$ is as follows. According to Theorem $B$ of [4] the transience of $\boldsymbol{X}_{-|\boldsymbol{W}|}^{0}$ (and consequently of $\boldsymbol{X}_{-|W|}$ ) follows if one proves that, for almost all Brownian environments $W$,

$$
\int_{0}^{\infty} e^{-|W(r \theta)|} r^{-d+1} d r<\infty
$$

for $\theta$ belonging to some subset (which may depend on $W$ ) of $S^{d-1}$ with a positive uniform measure. But this can be proved by showing that the expectation (with respect to $P$ ) of the left hand side of (2. 3) is finite for each fixed $\theta$.

\section{References}

[1] Th. Brox: A one-dimensional diffusion process in a Wiener medium. Ann. Probab., 14, 1206-1218 (1986).

[2] M. Fukushima: On recurrence criteria in the Dirichlet space theory. Local Times to Global Geometry, Control and Physics (ed. Elworthy). Research Notes in Math., Series 150, Longman (1987).

[3] M. Fukushima, S. Nakao and M. Takeda: On Dirichlet forms with random data recurrence and homogenization. Stochastic Processes - Mathematics and Physics II (eds. S. Albeverio, Ph. Blanchard and L. Streit). Lect. Notes in Math., vol. 1250, Springer-Verlag (1987).

[4] K. Ichihara: Some global properties of symmetric diffusion processes. Publ. RIMS, Kyoto Univ., 14, 441-486 (1978).

[ 5 ] K. Itô: On the ergodicity of a certain stationary process. Proc. Imp. Acad., 20, $54-55$ (1944). 
[6] P. Lévy: Processus Stochastiques et Mouvement Brownien. Gauthier-Villars, Paris (1948).

[ 7 ] P. Mathieu: Zero white noise limit through Dirichlet forms. Application to diffusions in a random medium (1992) (preprint).

[8] J. Nash: Continuity of solutions of parabolic and elliptic equations. Amer. J. Math., 80, 931-953 (1958).

[9] S. Schumacher: Diffusions with random coefficients. Contemporary Math. Particle Systems, Random Media and Large Deviations (ed. Durrett). vol. 41, pp. $351-356$ (1985).

[10] Y. G. Sinai: The limiting behavior of a one-dimensional random walk in a random medium. Theor. Probab. Appl., 27, 256-268 (1982).

[11] H. Tanaka: Limit theorem for one-dimensional diffusion process in Brownian environment. Stochastic Analysis (Proc. Japanese-French Seminar, Paris 1987). Lect. Notes in Math., vol. 1322, Springer-Verlag, pp. 156-172. 
